\title{
Variables underlying the recognition of random shapes'
}

HERBERT J. CLARK, ${ }^{2}$ AEROSPACE MEDICAL RESEARCH LABORATORIES RONALD L. KNOLL, BEHAVIOR RESEARCH LABORA TORY, ANTIOCH COLLEGE

Previous studies have shown that shape association value (percentage of Ss making an association to a shape) is a strong determining factor in shape recognition. In this study, the physical characteristics of shapes of high and low association value were compared. The intent was to uncover general physical characteristics of shape which differentiate shapes of high association value from shapes of low association value, and, thus, shapes accurately recognized from those not so accurately recognized. Comparisons were made on the basis of 12 factor scores per shape derived from an extensive analysis of 22 physical measures of shape. Results were that none of the factors differentiated between the two classes of shapes. Conclusions were that the physical measures may have been insensitive to perceptually conspicuous features in the perimeters of the shapes, and that associations to a shape are probably too varied in content to expect a few general physical measures to underlie those varied associations.

Recent studies have shown that an O's ability to make an association to a shape is a strong determining factor in shape recognition. For example, in a study that was published a few years ago (Clark, 1965), groups of Ss observed 10 simple or 10 complex shapes of high or low association value, and later attempted to recognize them in a forced-choice recognition test. Figures 1 and 2 show the shapes used in that study. All these shapes were selected from a set of 180 uncurved shapes constructed by Vanderplas and Garvin (1959), who used Attneave and Arnoult's (1956) Method 1 for generating random shapes.

Note that in each figure, the shapes are classified as being of

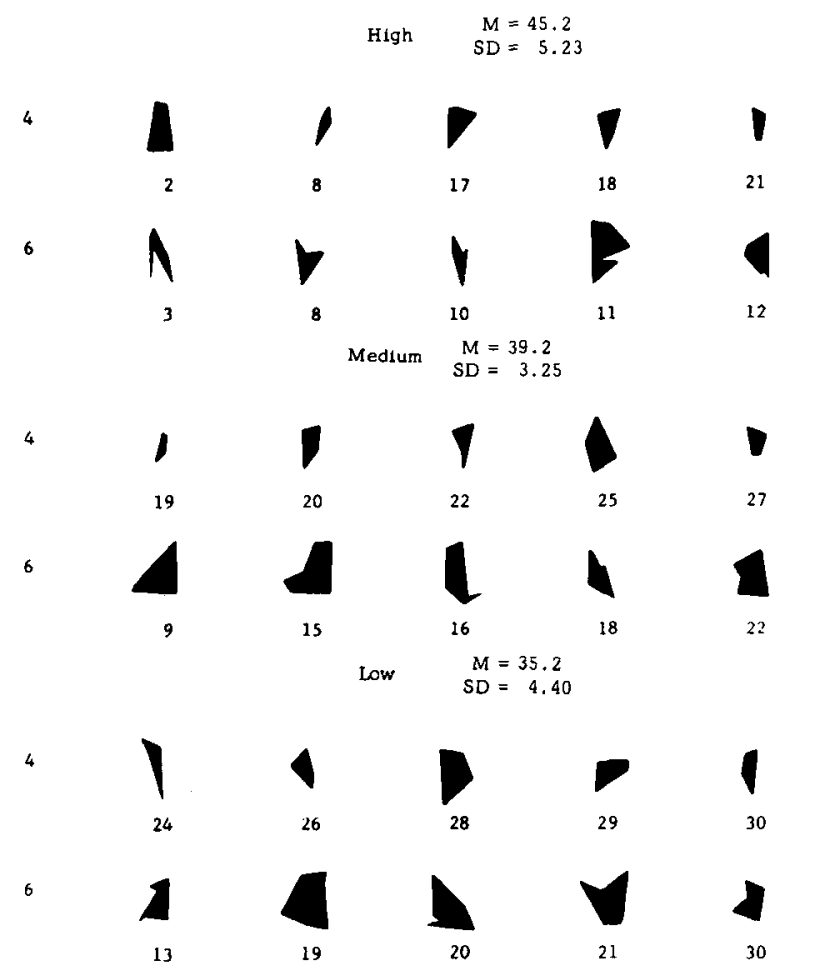

Fig. 1. Simple shapes of high, medium, and low association value. high, medium, or low association value. This measure is defined (Vanderplas \& Garvin, 1959) as the percentage of 50 Ss able to make an association to a shape. The mean Vanderplas and Garvin association values and standard deviations for the 10 shapes in each of the three associative categories are shown directly to the right of the word "high," "medium," or "low" in the figures. The number to the left of each row of five shapes specifies the number of points plotted in constructing that shape. It denotes the level of shape complexity (Attneave, 1957). In Fig. 1, the shapes were constructed using 4 or 6 points and they are called simple shapes. In Fig. 2, the shapes were constructed using 16 or 24 points and they are called complex shapes. The number of points plotted usually equals the number of sides in the perimeter of the shape. The number directly under each shape is that shape's Vanderplas and Garvin identification number.

In the recognition study referred to, the S's first task was to view the 10 simple or the 10 complex shapes of high or low association value shown in the figures. Shapes were presented one at a time on a white projection screen, each for $0.5 \mathrm{sec}$, with a $3.5-\mathrm{sec}$ interval between projections. After a brief delay, Ss attempted to recognize these 10 shapes in a forced-choice recognition test. There were 10 recognition trials. In each trial, the Ss saw two shapes, one at a time, each for $0.5 \mathrm{sec}$ with a 3.5-sec interval between members of a pair. One member of the pair was a previously observed shape and the other was a distractor shape of the same complexity level. Distractor shapes were selected from the shapes of medium association value shown in Figs. 1 and 2 . The S's task was to write the number " 1 " or " 2 " designating whether the first or second

$$
\text { High } \quad \begin{aligned}
M & =45.2 \\
\mathrm{SD} & =5.00
\end{aligned}
$$

16

(16)

24

2

16

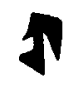

24

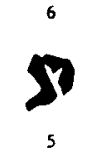

16

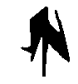

24

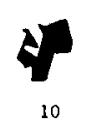

7
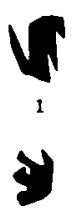

2

6

5

10
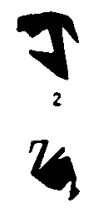

3

Medium

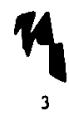

3

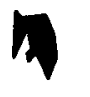

8

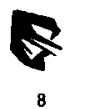

8

$$
\text { Low }
$$

15

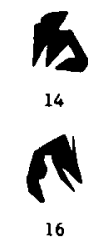

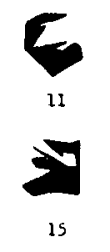
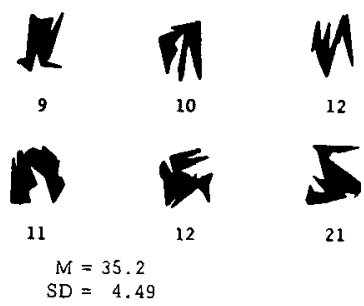

12
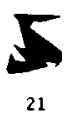

Fig. 2. Complex shapes of high, medium, and low association value. 
shape seen was a previously observed shape. This was done during a 7.5-sec interval between trials. Results of this study were that fewer errors of recognition were made for shapes of high association value than for shapes of low association value and that simple and complex shapes were recognized equally well.

Other studies from our laboratory have corroborated these results and have extended their generalizability. In one study, memory load was increased by having Ss remember 10 simple and 10 complex shapes of both high and low association value, instead of 10 simple or 10 complex shapes of either high or low association value. Exposure duration was $0.5 \mathrm{sec}$ as in the earlier study, but observed shapes were paired with distractor shapes of the opposite complexity level instead of the same complexity level as before. Results were the same; shapes of high association value were recognized more accurately than shapes of low association value, and simple and complex shapes were recognized equally well. In a replication and extension of this study (Clark, 1968), the effects of exposure duration were examined. One group viewed each shape for $0.50 \mathrm{sec}$, and another group viewed each shape for $0.25 \mathrm{sec}$. The effects of association value and complexity were still the same; the effects of exposure duration were insignificant.

Ellis, Muller, and Tosti (1966) have also established that association value has an incremental effect on shape recognition. They point out that perceptual performance depends upon the meaningfulness of the stimuli only to the degree that such stimuli readily elicit a single association, and that additional associations do not contribute to further gain in perceptual performance. In addition, Ellis and Homan (1968) have shown that E-supplied verbal labels enhance recognition performance.

All of these studies demonstrate that an O's ability to make an association to a shape is a strong determining factor in shape recognition. In the present study the investigation of association value was continued by comparing the physical characteristics of shapes of high and low association value. The intent was to determine if there are general physical characteristics of shape which differentiate shapes of high association value from shapes of low association value, and, thus, shapes accurately recognized from those not so accurately recognized.

\section{Shapes Compared}

\section{METHOD}

The shapes compared were those in Figs. 1 and 2. The complex shapes of high association value were compared with the complex shapes of low association value, and the simple shapes of high association value were compared with the simple shapes of low association value.

\section{Selection and Calculation of the Physical Measures}

Comparisons were made on the basis of 12 factor scores per shape. These scores were obtained from a factor analysis of 12 physical measures of shape derived from 22 other physical measures. The details of the procedures used in calculating all of these measures are described in a report by Knoll and Clark (1968); therefore, we will only summarize the measurement procedures.

The first step was to select 22 physical measures, most of which have been used in the past (Amoult, 1960; Attneave, 1957; Brown \& Owen, 1967; Stenson, 1966; Stilson, 1966; Vanderplas et al, 1965; Zusne, 1965). These 22 measures are described by Knoll and Clark (1968). Next, each of the 22 measures was taken on four sets of 30 shapes each. These were sets of 4, 6-, 16-, and 24-sided shapes, respectively, and were the Vanderplas and Garvin (1959) sets from which the shapes in Figs. 1 and 2 were selected. The complete sets were measured, instead of just the shapes in Figs. 1 and 2, to provide a more representative sample for study.

The technique employed in measuring the shapes was as follows. Each shape was enlarged photographically and the coordinates of the vertices were determined by overlaying the photographs with graph paper. These coordinates were then punched on IBM cards. Finally, the physical measurements were calculated using a computer program written by Knoll and Stenson (1968). This program can be used to generate and/or analyze random shapes.

Based on examination of the correlations and factor structure of the 22 measures, a reduced set of 12 measures for the 120 shapes was derived. These measures appeared to adequately describe the shapes, and they were nearly orthogonal to each other.

The last step was to factor analyze these 12 measures and then calculate factor scores for each of the 120 shapes. Results of a principal-axes factor analysis on the 12 measures are shown in Table 1. Twelve factors, A through $\mathbf{L}$, were rotated. Only loadings greater than .30 are included in the table, and each loading is rounded to two figures.

Mathematical symbols for the 12 physical measures are shown in the far left column of Table 1. You will note that each factor in Table 1 can be adequately described in terms of the physical measure having the highest loading on that factor. The 12 sets of factor scores (rather than the 12 physical measures) were used to compare the groups of shapes because the factor scores are more nearly orthogonal to each other (even for a subgroup) than the physical measures. Thus, a clearer interpretation of the sources of variation is permitted.

Definitions of the 12 physical measures, and thus the 12 factors, are as follows:

(1) Area (A). The area of the form.

(2) Compactness. The length of the perimeter divided by the square root of the area $\left(P / A^{1 / 2}\right)$.

(3) Orientation. The length from the positive $x$-axis to the positive first principal-axis, measured counterclockwise along a unit circle (with its center at the center of gravity of the

Table 1

Simple Structure Factor Loading Matrix for the 12 Physical Measures

\begin{tabular}{|c|c|c|c|c|c|c|c|c|c|c|c|c|}
\hline & A & B & $\mathrm{C}$ & $\mathrm{D}$ & $\mathrm{E}$ & $\mathrm{F}$ & $\mathrm{G}$ & $\mathrm{H}$ & $I$ & $\mathrm{~J}$ & $\mathrm{~K}$ & L \\
\hline $\begin{array}{l}\text { 1. Area } \\
\text { 2. P/A } / / 2 \\
\text { 3. Orientation } \\
\text { 4. } \mathrm{V}_{\mathrm{s}} / \mathrm{A} \\
\text { 5. } \mathrm{S}_{\mathrm{s}} / \mathrm{V}_{\mathrm{s}} 3 / 2 \\
\text { 6. } \mathrm{K}_{\mathrm{s}} / \mathrm{V}^{2} \\
\text { 7. } \mathrm{S}_{\mathrm{g}} / \mathrm{V}_{3 / 2} \\
\text { 8. } \mathrm{K}_{\mathrm{g}} / \mathrm{V}_{\mathrm{g}} \\
\text { 9. } \mu_{02} / \mu_{20} \\
\text { 10. } \mu_{30} / \mu_{20} / 2 \\
11 . \mu_{03} / \mu_{02} \\
12 . \mu_{03} / \mu_{30}\end{array}$ & $\begin{array}{l}-.34 \\
-.93 \\
- \\
\end{array}$ & $\begin{array}{r} \\
-.93 \\
-.40\end{array}$ & .91 & .98 & .99 & -.98 & .95 & .98 & $\begin{array}{r}.35 \\
-.93\end{array}$ & $\begin{array}{l} \\
-.94 \\
-.31\end{array}$ & .82 & $\begin{array}{l}-.33 \\
-.90\end{array}$ \\
\hline
\end{tabular}




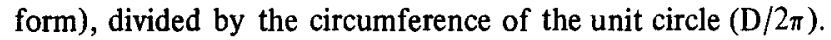

(4) Adjusted Straight Length Variance. The variance of the distribution of straight lengths in the perimeter of the shape divided by the area $\left(\mathrm{V}_{\mathrm{s}} / \mathrm{A}\right)$. This measure reflects the diversity in the lengths of straight segments in the perimeter of the shape. The reasons for dividing this measure and other adjusted measures by the area, or powers of moments, was to render the measures more independent of each other and independent of the size of the shape.

(5) Adjusted Straight Length Skewness. The skewness of the distribution of the straight lengths divided by the square root of the variance of the distribution of straight lengths raised to the third power $\left(S_{s} / V_{s}{ }^{3 / 2}\right)$.

(6) Adjusted Straight Length Kurtosis. The kurtosis of the distribution of straight lengths divided by the square of the variance of the distribution of the straight lengths $\left(\mathrm{K}_{\mathrm{s}} / \mathrm{V}_{\mathrm{s}}{ }^{2}\right)$.

(7) Adjusted Skewness of Internal Angles. The skewness of the distribution of the internal angles divided by the square root of the variance of the distribution of the internal angles raised to the third power $\left(S_{g} / V_{g}{ }^{3 / 2}\right)$.

(8) Adjusted Kurtosis of Internal Angles. The kurtosis of the distribution of internal angles divided by the square of the variance of the distribution of internal angles $\left(\mathrm{K}_{\mathrm{g}} / \mathrm{V}_{\mathrm{g}}{ }^{2}\right)$.

(9) Elongation. The area variance along the second principal-axis divided by the area variance along the first principal-axis $\left(\mu_{02} / \mu_{20}\right)$. As a form approaches a straight line, this measure approaches zero. As the form approximates a circle, it approaches 1 .

(10) First Principal-Axis Symmetry. The area skewness along the first principal axis divided by the square root of the third power of the area variance along the first principal-axis $\left(\mu_{30} / \mu_{20}{ }^{3 / 2}\right)$. A form with a teardrop shape has a high value on this measure while an ellipse has a zero value.

(11) Second Principal-Axis Symmetry. The area skewness along the second principal-axis divided by the square root of the third power of the area variance along the second principal-axis $\left(\mu_{03} / \mu_{02} 3 / 2\right)$.

(12) Two-Way Symmetry. The area skewness along the second principal-axis divided by the area skewness along the first principal-axis $\left(\mu_{0_{3}} / \mu_{30}\right)$.

\section{Statistical Analysis}

The 10 simple shapes of high association value were compared with the 10 simple shapes of low association value (see Fig. 1), and the 10 complex shapes of high association value were compared with the 10 complex shapes of low association value (see Fig. 2). Comparisons were made using the Mann-Whitney U Test. It was used because the sample sizes were small, and because the test is theoretically insensitive to the distribution of scores in the population.

To supplement the U-Test analysis, a correlational analysis was also performed. All four sets of Vanderplas and Garvin shapes, from which the shapes in Figs. 1 and 2 were selected, were included in this analysis. Within each set of 30 shapes, each of the 12 sets of factor scores was correlated with association value. Thus, within each set, 12 correlations were calculated, and each correlation was based on 30 pairs of measures.

\section{RESULTS AND DISCUSSION}

None of the 24 U-Tests was statistically significant. The 12 factors did not differentiate between shapes of high and low association value for either simple or complex shapes. Results of the correlational analysis were similar. None of the correlations was sufficiently high to suggest a reliable relationship between any single factor, or any group of factors, and association value. The correlations are shown in Table 2 . The per cent of variance in association value explained by the 12 factors is $30 \%$. This was determined by correlating the
Table 2

Correlations Between Association Value and Factor Scores for $4,6,16$, and 24 Point Shapes

\begin{tabular}{lrrrr}
\hline Factors & \multicolumn{1}{c}{4} & \multicolumn{1}{c}{6} & \multicolumn{1}{c}{16} & \multicolumn{1}{l}{24} \\
\hline A & -.14 & -.11 & .33 & -.05 \\
B & -.36 & .24 & .04 & .37 \\
C & .16 & -.20 & -.17 & -.24 \\
D & -.41 & .09 & -.09 & -.23 \\
E & -.19 & .09 & .14 & -.10 \\
F & -.06 & -.44 & -.08 & -.04 \\
G & .29 & .16 & .15 & -.23 \\
H & .05 & -.43 & .10 & .06 \\
I & -.29 & -.24 & .23 & -.19 \\
J & .02 & .38 & .23 & -.18 \\
K & .06 & -.07 & .01 & -.30 \\
L & .05 & .42 & .09 & -.19 \\
\hline
\end{tabular}

association values for the 120 shapes with the corresponding sets of factor scores for the 120 shapes and then squaring and summing these 12 correlations to obtain the per cent variance explained. This procedure was possible because the factor scores between factors for the entire set of 120 shapes are orthogonal to each other.

The failure to obtain a significant relationship between association value and our carefully derived physical measures of shape may be interpreted in various ways. From one point of view it may be concluded that the physical measures selected for study were inappropriate, and that the possibility remains of eventually finding physical characteristics which underlie association value as defined in this study. For example, it is possible that Ss make a majority of associations to perceptually conspicuous portions of the perimeter of the shape rather than to general characteristics of the whole shape. If so, our measures were inappropriate. None gave particular emphasis to salient characteristics of the perimeter. Relevant to this point of view are the results of Zusne (1965). He has pointed out that the physical measures he has used in discrimination studies have not given sufficient weight to the perceptually conspicuous portions of the perimeter. Therefore, he recommends weighting these parts of the perimeter more than its other parts. The problem with this approach is determining which parts of the perimeter should be classified as "salient."

From another point of view, it may be argued that association value, as defined in this study, should not necessarily correlate with any general or specific physical characteristics of shape. The thesis of this viewpoint is that associations are too varied in content to expect a few physical measures to underlie all of them. Data that we have collected indicate that associations to shapes are, indeed, often varied. Ten people may give 10 different associations to the same shape. Perhaps it is unlikely that only a few measures of shape underlie all these associations. Possibly, a different measure of association value, which accounts for the variety among associative responses, would be more likely to correlate with physical measures of shape. A major problem in deriving this new measure of assocaition value (which we would want to correlate with recognition accuracy) is determining which associative responses should be classified as "different" from each other.

\section{REFERENCES}

ARNOULT, M. D. Prediction of perceptual responses from structural characteristics of the stimulus. Perceptual \& Motor Skills, 1960, 11, 261-268.

ATTNEAVE, F. Physical determinants of the judged complexity of shapes. Journal of Experimental Psy chology, 1957, 53, 221-227.

ATTNEAVE, F., \& ARNOULT, M. D. The quantitative study of shape and pattern perception. Psychological Bulletin, 1956, 53, 452-471. 
BROWN, D. R., \& OWEN, D. H. The metrics of visual form: Methodological dyspepsia. Psychological Bulletin, 1967, 68, 4, 243-259.

CLARK, H. J. Recognition memory for random shapes as a function of complexity, association value, and delay. Joumal of Experimental Psychology, 1965, 69, 590-595.

CLARK, H. J. Random shape recognition at brief exposure durations. Psychonomic Science, 1968, 11, 351-352.

ELLIS, H. C., \& HOMAN, L. E. Implicit verbal responses and the transfer of stimulus predifferentiation. Journal of Experimental Psychology, $1968,76,486-489$.

ELLIS, H. C., MULLER, D. G., \& TOSTI, D. T. Stimulus meaning and complexity as factors in the transfer of stimulus predifferentiation. Joumal of Experimental Psychology, 1966, 71, 629-633.

KNOLL, R. L., \& CLARK, H. J. Physical characteristics and the factor structure of a selected set of random shapes. Aerospace Medical Research Laboratories Technical Report, in press.

KNOLL, R. L., \& STENSON, H. H. A computes program to construct and measure random forms. Perception \& Psychophysics, 1968, 3, 311-316.

STENSON, H. H. The physical factor structure of random forms and their judged complexity. Perception \& Psychophysics, 1966, 1, 303-310.

STILSON, D. W. A psychophysical investigation of triangular form. American Journal of Psychology, 1966, 79, 258-264.
VANDERPLAS, J. M., \& GARVIN, E. A. The association value of random shapes. Journal of Experimental Psychology, 1959, 57, 147-154.

VANDERPLAS, J. M., SANDERSON, W. A., \& VANDERPLAS, J. N. Statistical and associational characteristics of 1100 random shapes. Perceptual \& Motor Skills, 1965, 21, 414.

ZUSNE, L. Moments of area and of the perimeter of visual form as predictors of discrimination performance. Journal of Experimental Psychology, 1965, 69, 213-220.

\section{NOTES}

1. This paper has been identified by the Aerospace Medical Research Laboratories as AMRL-TR-68-149. Further reproduction is authorized to satisfy the needs of the U.S. Government. The research was performed in part by personnel of the Aerospace Medical Research Laboratories and in part by personnel of the Behavior Research Laboratory, Antioch College, under Contract F33615-67-C-1280. The report was presented at the 25th meeting of the Aerospace Medical Panel, Advisory Group for Aerospace Research and Development, NATO, which was held in London, England in October, 1968.

2. Address: Human Resources Laboratory, Wright-Patterson Air Force Base, Ohio 45433.

(Accepted for publication November $5,1968$. 